\title{
Clinical Medicine \& Research
}

\section{Subject Index: Volume 4, 2006 \\ March-December 2006}

\author{
Volume 4, Number 1, March 2006, Pages 1-96 \\ Volume 4, Number 2, June 2006, Pages 97-159 \\ Volume 4, Number 3, September 2006, Pages 160-244 \\ Volume 4, Number 4, December 2006, Pages 245-364
}

A

ABO blood-group system

Universal pathogen-reduced plasma in elective open-heart surgery and liver resection, 209

\section{Acinetobacter}

The use of intravenous and aerosolized polymyxins for the treatment of infections in critically ill patients: A review of the recent literature, 138

\section{Actin cytoskeleton}

Hereditary hemorrhagic telangiectasia, a vascular dysplasia affecting the TGF- $\beta$ signaling pathway, 66

\section{Alcohol}

Gender, treatment and self-help in remission from alcohol use disorders, 163

\section{Alcohol consumption}

Do women change their drinking behaviors while trying to conceive? An opportunity for preconception counseling, 97

\section{Alcoholics Anonymous}

Gender, treatment and self-help in remission from alcohol use disorders, 163

\section{ALK-1}

Hereditary hemorrhagic telangiectasia, a vascular dysplasia affecting the TGF- $\beta$ signaling pathway, 66

\section{Allelic association}

Innate immunity SNPs are associated with risk for severe sepsis after burn injury, 250

\section{Amoebicidal}

Amoebicidal activity of milk, apo-lactoferrin, slgA and lysozyme, 106

\section{Anemia}

Megaloblastic anemia and other causes of macrocytosis, 236

\section{Aneuploidy}

The emerging role of pituitary tumor transforming gene in tumorigenesis, 130

\section{Angiogenesis}

Hereditary hemorrhagic telangiectasia, a vascular dysplasia affecting the TGF- $\beta$ signaling pathway, 66

The emerging role of pituitary tumor transforming gene in tumorigenesis, 130
Ankylosing spondylitis

Cytokines and chemokines in uveitis - Is there a correlation with clinical phenotype? 294

\section{Antiendomysial antibodies}

The prevalence of growth hormone deficiency and celiac disease in short children, 180

\section{Apo E}

Genetic diagnosis and testing in clinical practice, 123

\section{Apo-lactoferrin}

Amoebicidal activity of milk, apo-lactoferrin, slgA and lysozyme, 106

Applanation tonometry

Nonlinear dynamics of blood pressure variability after caffeine consumption, 114

\section{Approximate entropy}

Nonlinear dynamics of blood pressure variability after caffeine consumption, 114

\section{Aqueous humor}

Cytokines and chemokines in uveitis - Is there a correlation with clinical phenotype? 294

\section{Arginase}

Recent advances in understanding endothelial dysfunction in atherosclerosis, 53

\section{Arginine}

Growth hormone stimulation tests in the differential diagnosis of Parkinson's disease, 322

\section{Arrhythmia}

Dysregulation of cell adhesion proteins and cardiac arrhythmogenesis, 42

\section{Asymptomatic hepatomegaly}

Asymptomatic hepatomegaly with elevated serum transaminases - Diagnosis? 336

\section{Atherosclerosis}

Recent advances in understanding endothelial dysfunction in atherosclerosis, 53

\section{Atrial systole}

Rene Theophile Hyacinthe Laënnec (1781-1826) - The man behind the stethoscope, 230 
Atypical parkinsonian disorders

Differential diagnosis of Parkinson's disease: A new blood test? 246

\section{B}

\section{Bacillus Calmette-Guérin}

Comparison of an in-house and a commercial RD1-based ELISPOT-IFN- $\gamma$ assay for the diagnosis of Mycobacterium tuberculosis infection, 266

\section{Barrett's esophagus}

Esophageal adenocarcinoma arising from Barrett's dysplasia: A case report of double occurrence and prolonged survival after chemotherapy, 184

\section{Behçet's disease}

Skewed Th1 responses caused by excessive expression of Txk, a member of Tec family tyrosine kinases, in patients with Behçet's disease, 147

Cytokines and chemokines in uveitis - Is there a correlation with clinical phenotype? 294

$\mathrm{BH}_{4}$ Recent advances in understanding endothelial dysfunction in atherosclerosis, $\mathbf{5 3}$

Black death

Dissecting plague, 161

\section{Bleomycin}

An armamentarium of wart treatments, $\mathbf{2 7 3}$

\section{Blood transfusion}

Universal pathogen-reduced plasma in elective open-heart surgery and liver resection, 209

\section{Body mass index}

Assessment of female participation in an employee 20-week walking incentive program at Marshfield Clinic, a large multispecialty group practice, 256

\section{BRCA}

Genetic diagnosis and testing in clinical practice, 123

\section{Burns}

Innate immunity SNPs are associated with risk for severe sepsis after burn injury, 250

\section{C}

\section{Cadherin}

Dysregulation of cell adhesion proteins and cardiac arrhythmogenesis, 42

\section{Caffeine}

Nonlinear dynamics of blood pressure variability after caffeine consumption, 114

\section{CAMP strains}

Imbalanced distribution of Plasmodium falciparum EBA-175 genotypes related to clinical status in children from Bakoumba, Gabon, 7

\section{Cancer gene therapy}

Gene therapy for cancer treatment: Past, present and future, 218
Cantharidin

An armamentarium of wart treatments, $\mathbf{2 7 3}$

Cardiac enzymes

Cardiac enzymes, renal failure and renal transplantation, $\mathbf{7 9}$

Cardiac neoplasms

The role of echocardiography in diagnosing space-occupying lesions of the heart, 22

\section{Cardiac troponins}

Cardiac enzymes, renal failure and renal transplantation, $\mathbf{7 9}$

Carpal tunnel syndrome

Jules Tinel (1879-1952) and Paul Hoffmann (1884-1962), 85

CD14

Innate immunity SNPs are associated with risk for severe sepsis after burn injury, 250

\section{Celiac disease}

The prevalence of growth hormone deficiency and celiac disease in short children, 180

\section{Cell cycle}

The emerging role of pituitary tumor transforming gene in tumorigenesis, 130

Cerebral infarction

The Siblings With Ischemic Stroke Study (SWISS): A progress report, 12

CFP-10

Comparison of an in-house and a commercial RD1-based ELISPOT-IFN- $\gamma$ assay for the diagnosis of Mycobacterium tuberculosis infection, 266

Chaos

Nonlinear dynamics of blood pressure variability after caffeine consumption, 114

\section{Charcot-Marie-Tooth}

Genetic diagnosis and testing in clinical practice, 123

\section{Chemokines}

Cytokines and chemokines in uveitis - Is there a correlation with clinical phenotype? 294

\section{Clinical Malaria}

Analysis of human antibodies to erythrocyte binding antigen 175 peptide 4 of Plasmodium falciparum, 1

Clonidine

Growth hormone stimulation tests in the differential diagnosis of Parkinson's disease, $\mathbf{3 2 2}$

\section{Coffee}

Nonlinear dynamics of blood pressure variability after caffeine consumption, 114

\section{Colistin}

The use of intravenous and aerosolized polymyxins for the treatment of infections in critically ill patients: A review of the recent literature, 138 
Computed tomography

Asymptomatic hepatomegaly with elevated serum

transaminases - Diagnosis? $\mathbf{3 3 6}$

\section{Computerized axial tomography}

Gastrointestinal stromal tumor with Dieulafoy lesion: A novel association, 228

\section{Connexin}

Dysregulation of cell adhesion proteins and cardiac

arrhythmogenesis, 42

\section{Coronary calcification}

Lipid-rich plaque masquerading as a coronary thrombus, 119

\section{Cost-effectiveness}

Universal pathogen-reduced plasma in elective open-heart surgery and liver resection, 209

\section{Cryotherapy}

An armamentarium of wart treatments, 273

\section{Cytokines}

Skewed Th1 responses caused by excessive expression of $\mathrm{Txk}$, a member of Tec family tyrosine kinases, in patients with Behçet's disease, 147

Cytokines in uveitis, 248

Cytokines and chemokines in uveitis - Is there a correlation with clinical phenotype? 294

\section{D}

Deep brain stimulation

Differential diagnosis of Parkinson's disease: A new blood test? 246

Degenerative health outcomes

Determinants of early life leptin levels and later life degenerative outcomes, 326

\section{Detrended fluctuation analysis}

Nonlinear dynamics of blood pressure variability after caffeine consumption, 114

\section{Diagnostic imaging}

The role of echocardiography in diagnosing space-occupying lesions of the heart, 22

\section{Dienga, Gabon}

Submicroscopic Plasmodium falciparum infections before and after sulfadoxine-pyrimethamine and artesunate association treatment in Dienga, Southeastern Gabon, 175

\section{Dieulafoy lesion}

Gastrointestinal stromal tumor with Dieulafoy lesion: A novel association, 228

\section{Differential diagnosis}

Differential diagnosis of Parkinson's disease: A new blood test? 246

\section{Diphencyprone}

An armamentarium of wart treatments, 273

DNA

Genetic diagnosis and testing in clinical practice, 123
Dyspepsia

Managing dyspepsia in a primary care setting, $\mathbf{3 3 7}$

\section{Dysplasia}

Esophageal adenocarcinoma arising from Barrettís dysplasia: A case report of double occurrence and prolonged survival after chemotherapy, 184

\section{$E$}

Early life

Determinants of early life leptin levels and later life degenerative outcomes, 326

\section{EBA-175 peptide 4}

Analysis of human antibodies to erythrocyte binding antigen 175 peptide 4 of Plasmodium falciparum, 1

\section{Echocardiography}

The role of echocardiography in diagnosing space-occupying lesions of the heart, 22

\section{Elevated serum transaminases}

Asymptomatic hepatomegaly with elevated serum transaminases - Diagnosis? 336

\section{ELISPOT}

Comparison of an in-house and a commercial RD1-based ELISPOT-IFN- $\gamma$ assay for the diagnosis of Mycobacterium tuberculosis infection, 266

\section{Endemic area}

Submicroscopic Plasmodium falciparum infections before and after sulfadoxine-pyrimethamine and artesunate association treatment in Dienga, Southeastern Gabon, 175

\section{Endoglin}

Hereditary hemorrhagic telangiectasia, a vascular dysplasia affecting the TGF- $\beta$ signaling pathway, 66

\section{Endoscopy}

Managing dyspepsia in a primary care setting, 337

\section{Endothelial cells}

Hereditary hemorrhagic telangiectasia, a vascular dysplasia affecting the TGF- $\beta$ signaling pathway, 66

\section{eNOS}

Recent advances in understanding endothelial dysfunction in atherosclerosis, $\mathbf{5 3}$

\section{Entamoeba histolytica}

Amoebicidal activity of milk, apo-lactoferrin, slgA and lysozyme, 106

\section{Entrapment neuropathy}

Jules Tinel (1879-1952) and Paul Hoffmann (1884-1962), 85

\section{Erythrocyte binding antigen $175 \mathrm{kDa}$}

Imbalanced distribution of Plasmodium falciparum EBA-175 genotypes related to clinical status in children from Bakoumba, Gabon, 7

\section{ESAT-6}

Comparison of an in-house and a commercial RD1-based ELISPOT-IFN- $\gamma$ assay for the diagnosis of Mycobacterium tuberculosis infection, 266 
Esophageal adenocarcinoma

Esophageal adenocarcinoma arising from Barrett's dysplasia: A case report of double occurrence and prolonged survival after chemotherapy, 184

\section{Esophagogastroduodenoscopy}

Gastrointestinal stromal tumor with Dieulafoy lesion: A novel association, 228

\section{Eye diseases}

Cytokines in uveitis, 248

\section{$\mathbf{F}$}

Factor V Leiden

Genetic diagnosis and testing in clinical practice, 123

FCR-3 strains

Imbalanced distribution of Plasmodium falciparum EBA-175 genotypes related to clinical status in children from

Bakoumba, Gabon, 7

Female

Assessment of female participation in an employee 20-week walking incentive program at Marshfield Clinic, a large multispecialty group practice, 256

Fetal outcomes

Lupus and pregnancy: Complex yet manageable, $\mathbf{3 1 0}$

\section{Fractal}

Nonlinear dynamics of blood pressure variability after caffeine consumption, 114

Cytokines and chemokines in uveitis - Is there a correlation with clinical phenotype? 294

Fuch's heterochromic cyclitis

Cytokines and chemokines in uveitis - Is there a correlation with clinical phenotype? 294

\section{G}

\section{Gap junctions}

Dysregulation of cell adhesion proteins and cardiac arrhythmogenesis, $\mathbf{4 2}$

\section{Gastroesophageal reflux disease}

Esophageal adenocarcinoma arising from Barrett's dysplasia: A case report of double occurrence and prolonged survival after chemotherapy, 184

\section{Gastrointestinal stromal tumor}

Gastrointestinal stromal tumor with Dieulafoy lesion: A novel association, 228

\section{Gender}

Gender, treatment and self-help in remission from alcohol use disorders, 163

\section{Gene transfer}

Gene therapy for cancer treatment: Past, present and future, $\mathbf{2 1 8}$

Genetic testing

Genetic diagnosis and testing in clinical practice, 123
Genomic comparison

Current trends in plague research: From genomics to virulence, 189

\section{Genomics}

Dissecting plague, 161

\section{Growth hormone}

Growth hormone stimulation tests in the differential diagnosis of Parkinson's disease, 322

Growth hormone deficiency

The prevalence of growth hormone deficiency and celiac disease in short children, 180

\section{Growth hormone test}

Differential diagnosis of Parkinson's disease: A new blood test? 246

\section{$\mathbf{H}$}

\section{Head and neck}

Postoperative radiotherapy for squamous cell carcinoma of the head and neck, 200

Help-seeking

Gender, treatment and self-help in remission from alcohol use disorders, 163

\section{Hereditary hemochromatosis}

Genetic diagnosis and testing in clinical practice, 123

Hereditary hemorrhagic telangiectasia

Hereditary hemorrhagic telangiectasia, a vascular dysplasia affecting the TGF- $\beta$ signaling pathway, 66

Hoffmann-Tinel sign

Jules Tinel (1879-1952) and Paul Hoffmann (1884-1962), 85

Household contacts

Comparison of an in-house and a commercial RD1-based ELISPOT-IFN- $\gamma$ assay for the diagnosis of Mycobacterium tuberculosis infection, 266

Human papilloma virus

An armamentarium of wart treatments, 273

Huntington's disease

Genetic diagnosis and testing in clinical practice, 123

\section{I}

IFN- $\gamma$

Comparison of an in-house and a commercial RD1-based ELISPOT-IFN- $\gamma$ assay for the diagnosis of Mycobacterium tuberculosis infection, 266

IL-6 Innate immunity SNPs are associated with risk for severe sepsis after burn injury, 250

\section{Imaging}

The role of echocardiography in diagnosing space-occupying lesions of the heart, $\mathbf{2 2}$ 
Imiquimod

An armamentarium of wart treatments, 273

\section{Immune response}

Analysis of human antibodies to erythrocyte binding antigen

175 peptide 4 of Plasmodium falciparum, 1

\section{Immunotherapy}

Gene therapy for cancer treatment: Past, present and future, 218

Infectious disease

Dissecting plague, 161

Inflammatory response

Cytokines in uveitis, 248

\section{Inhalation}

The use of intravenous and aerosolized polymyxins for the treatment of infections in critically ill patients: A review of the recent literature, 138

\section{Intensive care unit}

The use of intravenous and aerosolized polymyxins for the treatment of infections in critically ill patients: A review of the recent literature, 138

\section{Intercalated disc}

Dysregulation of cell adhesion proteins and cardiac arrhythmogenesis, 42

Intravascular ultrasound

Lipid-rich plaque masquerading as a coronary thrombus, 119

\section{Ischemic stroke}

The Siblings With Ischemic Stroke Study (SWISS): A progress report, 12

\section{$\mathbf{K}$}

\section{Klebsiella}

The use of intravenous and aerosolized polymyxins for the treatment of infections in critically ill patients: A review of the recent literature, 138

\section{$\mathbf{L}$}

\section{Laënnec's Cirrhosis}

Rene Theophile Hyacinthe Laënnec (1781-1826) - The man behind the stethoscope, 230

\section{L-arginine}

Recent advances in understanding endothelial dysfunction in atherosclerosis, $\mathbf{5 3}$

\section{Laser}

An armamentarium of wart treatments, 273

\section{Later life}

Determinants of early life leptin levels and later life degenerative outcomes, 326

\section{Leptin}

Determinants of early life leptin levels and later life degenerative outcomes, 326

\section{Lipid plaques and filling defects}

Lipid-rich plaque masquerading as a coronary thrombus, 119

\section{Liver resection}

Universal pathogen-reduced plasma in elective open-heart surgery and liver resection, 209

Lupus

Lupus and pregnancy: Complex yet manageable, $\mathbf{3 1 0}$

Lysozyme

Amoebicidal activity of milk, apo-lactoferrin, slgA and lysozyme, 106

\section{M}

\section{Macrocytosis}

Megaloblastic anemia and other causes of macrocytosis, 236

\section{Magnetic resonance}

The role of echocardiography in diagnosing space-occupying lesions of the heart, 22

\section{Malaria}

Interactions between natural killer cells, cortisol and prolactin in malaria during pregnancy, 33

\section{Management}

Managing dyspepsia in a primary care setting, 337

\section{Marfan syndrome}

Genetic diagnosis and testing in clinical practice,123

\section{Maternal outcomes}

Lupus and pregnancy: Complex yet manageable, $\mathbf{3 1 0}$

\section{Mediate auscultation}

Rene Theophile Hyacinthe Laënnec (1781-1826) - The man behind the stethoscope, $\mathbf{2 3 0}$

\section{Megaloblastic}

Megaloblastic anemia and other causes of macrocytosis, 236

\section{Melanoma}

Rene Theophile Hyacinthe Laënnec (1781-1826) - The man behind the stethoscope, 230

Milk

Amoebicidal activity of milk, apo-lactoferrin, slgA and lysozyme, 106

\section{Multidrug-resistant Gram-negative bacteria}

The use of intravenous and aerosolized polymyxins for the treatment of infections in critically ill patients: A review of the recent literature, 138

\section{Multiple system atrophy}

Growth hormone stimulation tests in the differential diagnosis of Parkinson's disease, 322

\section{Multiplex}

Cytokines and chemokines in uveitis - Is there a correlation with clinical phenotype? 294

\section{N}

\section{Natural killer cells}

Interactions between natural killer cells, cortisol and prolactin in malaria during pregnancy, $\mathbf{3 3}$ 


\section{Nebulization}

The use of intravenous and aerosolized polymyxins for the treatment of infections in critically ill patients: A review of the recent literature, 138

\section{Nonlinear dynamics}

Nonlinear dynamics of blood pressure variability after caffeine consumption, 114

\section{Nonulcerative dyspepsia}

Managing dyspepsia in a primary care setting, $\mathbf{3 3 7}$

\section{0}

\section{Oncolytic virotherapy}

Gene therapy for cancer treatment: Past, present and future, 218

\section{Open-heart surgery}

Universal pathogen-reduced plasma in elective open-heart surgery and liver resection, 209

\section{Outcome}

Gender, treatment and self-help in remission from alcohol use disorders, 163

\section{Oxidative stress}

Recent advances in understanding endothelial dysfunction in atherosclerosis, $\mathbf{5 3}$

\section{$\mathbf{P}$}

\section{Parkinson's disease}

Differential diagnosis of Parkinson's disease: A new blood test? 246

Growth hormone stimulation tests in the differential diagnosis of Parkinson's disease, 322

\section{Pathogen reduction}

Universal pathogen-reduced plasma in elective open-heart surgery and liver resection, 209

PCR

Submicroscopic Plasmodium falciparum infections before and after sulfadoxine-pyrimethamine and artesunate association treatment in Dienga, Southeastern Gabon, 175

\section{Pedigree research}

The Siblings With Ischemic Stroke Study (SWISS): A progress report, 12

\section{Percutaneous coronary intervention}

Lipid-rich plaque masquerading as a coronary thrombus, 119

\section{Physical activity}

Assessment of female participation in an employee 20-week walking incentive program at Marshfield Clinic, a large multispecialty group practice, 256

\section{Pituitary adenoma}

The emerging role of pituitary tumor transforming gene in tumorigenesis, 130

Plague

Dissecting plague, 161

\section{Plasmodium falciparum}

Analysis of human antibodies to erythrocyte binding antigen 175 peptide 4 of Plasmodium falciparum, 1

Imbalanced distribution of Plasmodium falciparum EBA-175 genotypes related to clinical status in children from

Bakoumba, Gabon, 7

Interactions between natural killer cells, cortisol and prolactin in malaria during pregnancy, 33

\section{Pneumonia}

The use of intravenous and aerosolized polymyxins for the treatment of infections in critically ill patients: A review of the recent literature, 138

Polymorphism

Innate immunity SNPs are associated with risk for severe sepsis after burn injury, 250

Polymyxin B

The use of intravenous and aerosolized polymyxins for the treatment of infections in critically ill patients: A review of the recent literature, 138

PPD

Comparison of an in-house and a commercial RD1-based ELISPOT-IFN- $\gamma$ assay for the diagnosis of Mycobacterium tuberculosis infection, 266

Preconception counseling

Do women change their drinking behaviors while trying to conceive? An opportunity for preconception counseling, 97

Predictive testing

Genetic diagnosis and testing in clinical practice, 123

\section{Pregnancy}

Interactions between natural killer cells, cortisol and prolactin in malaria during pregnancy, 33

Do women change their drinking behaviors while trying to conceive? An opportunity for preconception counseling, 97 Lupus and pregnancy: Complex yet manageable, $\mathbf{3 1 0}$

\section{Pressure waveform}

Nonlinear dynamics of blood pressure variability after caffeine consumption, 114

\section{Prevalence}

Submicroscopic Plasmodium falciparum infections before and after sulfadoxine-pyrimethamine and artesunate association treatment in Dienga, Southeastern Gabon, 175 The prevalence of growth hormone deficiency and celiac disease in short children, 180

\section{Proband}

The Siblings With Ischemic Stroke Study (SWISS): A progress report, 12

\section{Pseudomonas}

The use of intravenous and aerosolized polymyxins for the treatment of infections in critically ill patients: A review of the recent literature, 138

Public health

Dissecting plague, 161 
$\mathbf{R}$

Radiotherapy

Postoperative radiotherapy for squamous cell carcinoma of the head and neck, 200

\section{Recruitment}

The Siblings With Ischemic Stroke Study (SWISS): A

progress report, 12

Renal failure

Cardiac enzymes, renal failure and renal transplantation, $\mathbf{7 9}$

Renal transplantation

Cardiac enzymes, renal failure and renal transplantation, $\mathbf{7 9}$

\section{S}

\section{Sarcoidosis}

Cytokines and chemokines in uveitis - Is there a correlation with clinical phenotype? 294

\section{Sepsis}

Innate immunity SNPs are associated with risk for severe sepsis after burn injury, $\mathbf{2 5 0}$

\section{Short children}

The prevalence of growth hormone deficiency and celiac disease in short children, 180

$\operatorname{sig} A$

Amoebicidal activity of milk, apo-lactoferrin, slgA and lysozyme, 106

SNP

Innate immunity SNPs are associated with risk for severe sepsis after burn injury, 250

\section{Squamous cell carcinoma}

Postoperative radiotherapy for squamous cell carcinoma of the head and neck, 200

\section{Submicroscopic Plasmodium falciparum}

Submicroscopic Plasmodium falciparum infections before and after sulfadoxine-pyrimethamine and artesunate association treatment in Dienga, Southeastern Gabon, 175

\section{Sulfadoxine-pyrimethamine-artesunate}

Submicroscopic Plasmodium falciparum infections before and after sulfadoxine-pyrimethamine and artesunate association treatment in Dienga, Southeastern Gabon, 175

\section{SWISS}

The Siblings with Ischemic Stroke Study (SWISS): A progress report, 12

Systemic lupus erythematosus

Lupus and pregnancy: Complex yet manageable, $\mathbf{3 1 0}$

$\mathbf{T}$

TGF- $\beta$ pathway

Hereditary hemorrhagic telangiectasia, a vascular dysplasia affecting the TGF- $\beta$ signaling pathway, 66
Th I cell

Skewed Th1 responses caused by excessive expression of Txk, a member of Tec family tyrosine kinases, in patients with Behçet's disease, 147

Tibial nerve decompression

Jules Tinel (1879-1952) and Paul Hoffmann (1884-1962), 85

Tinel's sign

Jules Tinel (1879-1952) and Paul Hoffmann (1884-1962), 85

\section{TLR4}

Innate immunity SNPs are associated with risk for severe sepsis after burn injury, 250

TNF- $\alpha$

Innate immunity SNPs are associated with risk for severe sepsis after burn injury, 250

Tomography

The role of echocardiography in diagnosing space-occupying lesions of the heart, 22

\section{Treatment}

Gender, treatment and self-help in remission from alcohol use disorders, 163

\section{Tuberculin skin test}

Comparison of an in-house and a commercial RD1-based ELISPOT-IFN- $\gamma$ assay for the diagnosis of Mycobacterium tuberculosis infection, 266

\section{Tuberculosis}

Rene Theophile Hyacinthe Laënnec (1781-1826) - The man behind the stethoscope, $\mathbf{2 3 0}$

Comparison of an In-house and a commercial RD1-based ELISPOT-IFN- $\gamma$ assay for the diagnosis of Mycobacterium tuberculosis infection, 266

\section{Txk}

Skewed Th1 responses caused by excessive expression of Txk, a member of Tec family tyrosine kinases, in patients with Behçet's disease, 147

\section{$\mathbf{U}$}

\section{Universal ABO-independent plasma}

Universal pathogen-reduced plasma in elective open-heart surgery and liver resection, 209

\section{Uveitis}

Cytokines in uveitis, 248

Cytokines and chemokines in uveitis - Is there a correlation with clinical phenotype? 294

\section{V}

Vaccine

An armamentarium of wart treatments, 273

\section{Variability}

Nonlinear dynamics of blood pressure variability after caffeine consumption, 114 
Vascular dysplasia

Hereditary hemorrhagic telangiectasia, a vascular dysplasia affecting the TGF- $\beta$ signaling pathway, 66

\section{Ventilator-associated}

The use of intravenous and aerosolized polymyxins for the treatment of infections in critically ill patients: A review of the recent literature, 138

Ventricular systole

Rene Theophile Hyacinthe Laënnec (1781-1826) - The man behind the stethoscope, $\mathbf{2 3 0}$

\section{Virucidal}

An armamentarium of wart treatments, 273

\section{Virulence}

Dissecting plague, 161

Current trends in plague research: From genomics to virulence, 189

\section{Vogt-Koyanagi-Harada disease}

Cytokines and chemokines in uveitis - Is there a correlation with clinical phenotype? 294

\section{W}

\section{Walking}

Assessment of female participation in an employee 20-week walking incentive program at Marshfield Clinic, a large multispecialty group practice, 256

Wart

An armamentarium of wart treatments, $\mathbf{2 7 3}$

\section{$\mathbf{Y}$}

\section{Yersinia pestis}

Dissecting plague, 161

Yersinia spp.

Current trends in plague research: From genomics to virulence, 189 